\title{
Invariant *-products on coadjoint orbits and the Shapovalov pairing
}

\author{
A. Alekseev and A. Lachowska
}

Abstract. We give an explicit formula for invariant $*$-products on a wide class of coadjoint orbits. The answer is expressed in terms of the Shapovalov pairing for generalized Verma modules.

\section{Introduction}

The problem of constructing a $*$-product on a manifold $M$ with given Poisson structure was formulated in [BFFLS]. In the case of symplectic manifolds the existence of $*$ products was established in [DL] and in a more geometric fashion in [F]. For a general Poisson manifold the existence of $*$-products was proved in $[\mathrm{K}]$. While general existence results are now available, giving explicit formulas for $*$-products remains a difficult task. The first formula of this type was given by Moyal in $[\mathrm{M}]$ in the case of a constant Poisson bi-vector on $\mathbb{R}^{n}$. Further examples that one can consider are linear Poisson brackets on the dual $\mathfrak{g}^{*}$ of the Lie algebra $\mathfrak{g}$, and nondegenerate Poisson brackets on coadjoint orbits in $\mathfrak{g}^{*}$. The $*$-products on $\mathfrak{g}^{*}$ were constructed, for example, in [G]. It is a natural idea to construct $*$-products on coadjoint orbits by restriction from $\mathfrak{g}^{*}$. However, in [CGR] it was proved that for $\mathfrak{g}$ semisimple, a smooth $*$-product on $\mathfrak{g}^{*}$ does not restrict to coadjoint orbits.

Examples of $*$-products on some simple coadjoint orbits $\left(C \mathrm{P}^{n}\right.$ and symmetric spaces) can be found in $[\mathrm{BBEW}],[\mathrm{T}]$. We shall concentrate on constructing *-products on $M$ invariant with respect to the transitive $G$-action. In the case of $G=\mathrm{GL}(n)$ this problem has been addressed in [DM1]. Invariant $*$-products on minimal nilpotent coadjoint orbits of simple Lie groups were constructed in $[\mathrm{ABC}]$ and $[\mathrm{AB}]$. In these works the locality axiom (stating that the $*$-product should be defined by a bi-differential operator) is relaxed. In the case of $\mathfrak{g}$ semisimple constructions of *-products on coadjoint orbits of semisimple elements were suggested in [A] using the deformation quantization with separation of variables of [Ka], and in [DM2] using the methods of category theory.

Our main result is an explicit formula for invariant $*$-products on coadjoint orbits $G / H$, for which the corresponding Lie algebras $\mathfrak{g}$ and $\mathfrak{h}$ (or their complexifications) 
fit into a decomposition $\mathfrak{g}=\mathfrak{n}_{+} \oplus \mathfrak{h} \oplus \mathfrak{n}_{-}$(see Section 2.2 for the precise statement of assumptions). Examples include: the space $\mathbb{R}^{2 n}$ with constant nondegenerate Poisson bracket (a coadjoint orbit in the dual of the Heisenberg algebra), coadjoint orbits of semisimple elements in semisimple Lie algebras, as well as infinite dimensional examples such as coadjoint orbits in the dual to the Virasoro algebra. Our construction is motivated by the fusion techniques of [EV].

Acknowledgements. We received a lot of help from S. Parmentier, who participated in this project in its early stages. We are grateful to S. Gutt, P. Etingof, V. Rubtsov, D. Sternheimer, X. Tang, B. Tsygan and P. Xu for the interest in our work and for useful discussions. This work was supported in part by the Swiss National Science foundation and by the Erwin Schrödinger Institute for Mathematical Physics.

\section{Preliminaries}

In this section we formulate the problem of finding invariant $*$-products on homogeneous spaces, and we state the assumptions, which allow us to solve it in an explicit form.

2.1. Invariant *-products on homogeneous spaces. Let $M$ be a manifold. Recall that a $*$-product on $M$ is defined by a formal power series $B=\sum_{n=0}^{\infty} \hbar^{n} B_{n}$ in $\hbar$, where the coefficients are complex bi-differential operators on $M$ with $B_{0}=1$ and $B_{n} \in$ $\operatorname{Diff}^{2}(M)$. The $*$-product is then given by the formula $f * g:=f g+\sum_{n=1}^{\infty} \hbar^{n} B_{n}(f, g)$ for $f, g \in C^{\infty}(M)$. The main condition imposed on $B$ is that the $*$-product be associative, that is, $f *(g * h)=(f * g) * h$ for all $f, g, h \in C^{\infty}(M)$.

Let $G$ be a connected Lie group and let $H \subset G$ be a closed Lie subgroup of $G$. Denote the corresponding Lie algebras by $\mathfrak{g}$ and $\mathfrak{h}$, respectively. The quotient $M:=G / H$ carries a transitive action of $G$. There is an induced $G$-action on functions on $M$, and on differential and poly-differential operators. A $*$-product on $M$ is called invariant if it is defined by an invariant formal bi-differential operator $B$. That is, all bi-differential operators $B_{n}$ have to be $G$-invariant.

Recall that the space of invariant differential operators on $M=G / H$ can be expressed as follows: $\operatorname{Diff}_{G}(M)=(U \mathfrak{g} / U \mathfrak{g} \cdot \mathfrak{h})^{H}$. Here $U \mathfrak{g}$ is the universal enveloping algebra of $\mathfrak{g}, U \mathfrak{g} \cdot \mathfrak{h}$ is the left ideal generated by $\mathfrak{h} \subset \mathfrak{g} \subset U \mathfrak{g}$. The algebra $U \mathfrak{g}$ carries a natural adjoint action of $H \subset G$, and since $\operatorname{Ad}_{H}(\mathfrak{h})=\mathfrak{h}$, this action factors to the quotient $U \mathfrak{g} / U \mathfrak{g} \cdot \mathfrak{h}$. The $H$-invariant part $(U \mathfrak{g} / U \mathfrak{g} \cdot \mathfrak{h})^{H}$ has an algebra structure induced by the one of $U \mathfrak{g}$. In a similar fashion, the space of invariant $N$-differential operators on $G / H$ is given by

$$
\operatorname{Diff}_{G}^{N}(M)=\left((U \mathfrak{g} / U \mathfrak{g} \cdot \mathfrak{h})^{\otimes N}\right)^{H},
$$


where the invariant part is taken with respect to the diagonal $\mathrm{H}$-action on $(U \mathfrak{g} / U \mathfrak{g} \cdot \mathfrak{h})^{\otimes N}$. Formal bi-differential operators which define invariant $*$-products on $M$ take values in the space

$$
B \in\left((U \mathfrak{g} / U \mathfrak{g} \cdot \mathfrak{h})^{\otimes 2}\right)^{H}[[\hbar]] .
$$

Remark 2.1. As opposed to $\operatorname{Diff}_{G}(M)$, the space $\operatorname{Diff}_{G}^{N}(M)$ for $N>1$ has no natural algebra structure.

Let $\Delta: U \mathfrak{g} \rightarrow U \mathfrak{g} \otimes U \mathfrak{g}$ be the standard coproduct of $U \mathfrak{g}$. That is, $\Delta$ is an algebra homomorphism such that $\Delta(x)=1 \otimes x+x \otimes 1$ for all $x \in \mathfrak{g}$. Let $B, C \in \operatorname{Diff}_{G}^{2}(M)=$ $\left((U \mathfrak{g} / U \mathfrak{g} \cdot \mathfrak{h})^{\otimes 2}\right)^{H}$. Then the expressions $((\Delta \otimes 1) B)(C \otimes 1)$ and $((1 \otimes \Delta) B)(1 \otimes C)$ define unique elements of $\operatorname{Diff}_{G}^{3}(M)=\left((U \mathfrak{g} / U \mathfrak{g} \cdot \mathfrak{h})^{\otimes 3}\right)^{H}$. In more detail, let $\hat{B}, \hat{C} \in U \mathfrak{g} \otimes U \mathfrak{g}$ be representatives of the classes $B, C \in(U \mathfrak{g} / U \mathfrak{g} \cdot \mathfrak{h})^{\otimes 2}$. The classes of $((\Delta \otimes 1) \hat{B})(\hat{C} \otimes 1)$ and $((1 \otimes \Delta) \hat{B})(1 \otimes \hat{C})$ in $(U \mathfrak{g} / U \mathfrak{g} \cdot \mathfrak{h})^{\otimes 3}$ are $H$ invariant and independent of the choice of representatives.

The associativity of an invariant $*$-product defined by a formal bi-differential operator $B$ is expressed by the following equality of invariant formal 3-differential operators in $\left((U \mathfrak{g} / U \mathfrak{g} \cdot \mathfrak{h})^{\otimes 3}\right)^{H}[[\hbar]]$ :

$$
((\Delta \otimes 1) B)(B \otimes 1)=((1 \otimes \Delta) B)(1 \otimes B) .
$$

Remark 2.2. If $H$ is a connected Lie group one can replace the condition of $\mathrm{Ad}_{H^{-}}$ invariance of $B$ by an algebraic condition of invariance with respect to the adjoint action of $\mathfrak{h}$. It is also a natural context when the Lie algebra $\mathfrak{g}$ is infinite dimensional and the corresponding Lie group may not be available.

Remark 2.3. In the case of $\mathfrak{h}=0$, equation (1) was considered by Drinfeld in [Dr]; here a family of solutions of (1) was constructed in terms of the Campbell-Hausdorff series for the Lie algebra $\mathfrak{g}$.

2.2. Assumptions on $\mathfrak{g}$ and $\mathfrak{h}$. Examples. Here we list assumptions imposed on the Lie algebra $\mathfrak{g}$ and its Lie subalgebra $\mathfrak{h}$ which allow to construct explicit solutions of equation (1).

- The Lie algebra $\mathfrak{g}$ is $\mathbb{Z}$-graded, $\mathfrak{g}=\bigoplus_{i \in \mathbb{Z}} \mathfrak{g}_{i}$, such that each graded component has finite dimension. The adjoint action of $H$ on $\mathfrak{g}$ preserves this grading. Let $\mathfrak{h}=\mathfrak{g}_{0}$ and denote $\mathfrak{n}_{+}=\bigoplus_{i>0} \mathfrak{g}_{i}, \mathfrak{n}_{-}=\bigoplus_{i<0} \mathfrak{g}_{i}$

- There exists a character $\chi: \mathfrak{g}_{0} \rightarrow \mathbb{C}$ such that the pairing $\mathfrak{n}_{+} \times \mathfrak{n}_{-} \rightarrow \mathbb{C}$ defined by $u, v \mapsto \chi\left([u, v]_{0}\right)$ for $u \in \mathfrak{n}_{+}, v \in \mathfrak{n}_{-}$is nondegenerate. Here $x \mapsto x_{0}$ denotes the projection onto the zero graded component $\mathfrak{g}_{0}$. Such a character is called nonsingular. 
Remark 2.4. If $H$ is connected, the requirement that $H$ preserves the grading is automatically satisfied.

Remark 2.5. The second assumption implies that $\mathfrak{g}_{0} \neq 0$.

Example 2.6. Assume that $\mathfrak{g}$ is quadratic. That is, there is a nondegenerate $\operatorname{ad}(\mathfrak{g})$ invariant symmetric bilinear form $Q: \mathfrak{g} \times \mathfrak{g} \rightarrow \mathbb{C}$ of degree zero. In this case, $\mathfrak{g}_{0}$ is a finite dimensional quadratic Lie algebra with invariant bilinear form, the restriction of $Q$ to $\mathfrak{g}_{0}$. Let $\mathfrak{z}\left(\mathfrak{g}_{0}\right)$ be the center of $\mathfrak{g}_{0}$. Every element $z \in \mathfrak{z}\left(\mathfrak{g}_{0}\right)$ defines a character $\chi_{z}$ of $\mathfrak{g}_{0}, \chi_{z}(x)=Q(z, x)$. An element $z \in \mathfrak{z}\left(\mathfrak{g}_{0}\right)$ is called nonsingular if the map $(u, v) \mapsto \chi_{z}\left([u, v]_{0}\right) \in \mathbb{C}$ defines a nondegenerate pairing between the graded components $\mathfrak{g}_{i}$ and $\mathfrak{g}_{-i}$ for all $i$. If $\mathfrak{z}\left(\mathfrak{g}_{0}\right)$ is nonempty and contains a nonsingular element, then the set of such elements is Zariski open in $\mathfrak{z}\left(\mathfrak{g}_{0}\right)$.

Example 2.7. Let $\mathfrak{g}$ be a semisimple complex Lie algebra, $\mathfrak{h}$ be a Cartan subalgebra of $\mathfrak{g}, \Pi=\left\{\alpha_{i}\right\}_{i=1}^{\text {rank }(\mathfrak{g})}$ be (some choice of) the set of simple roots. The principal grading on $\mathfrak{g}$ is the unique grading such that $\mathfrak{h}=\mathfrak{g}_{0}$ and all root vectors corresponding to simple roots have degree $1, e_{\alpha_{i}} \in \mathfrak{g}_{1}$. Any regular character $\chi$ of $\mathfrak{h}$ defines a nondegenerate pairing $\mathfrak{n}_{+} \times \mathfrak{n}_{-} \rightarrow \mathbb{C}$.

This construction applies verbatim to any Kac-Moody algebra.

Example 2.8. Let $\mathfrak{g}$ be a semisimple complex Lie algebra, and let $z \in \mathfrak{g}$ be a semisimple element. Let $\mathfrak{g}_{z}$ be the centralizer of $z$ and let $\mathfrak{h}$ be a Cartan subalgebra of $\mathfrak{g}$ such that $z \in \mathfrak{h} \subset \mathfrak{g}_{z}$. Define the unique grading on $\mathfrak{g}$ such that $\mathfrak{g}_{0}=\mathfrak{g}_{z}$ and for all simple roots in $S_{z}:=\left\{\alpha_{i}, e_{\alpha_{i}} \notin \mathfrak{g}_{z}\right\}$ one has $e_{\alpha_{i}} \in \mathfrak{g}_{1}$. The Lie algebra $\mathfrak{p}_{+}=\mathfrak{g}_{0} \oplus \mathfrak{n}_{+}$is a parabolic subalgebra of $\mathfrak{g}$ with the Levi subalgebra $\mathfrak{g}_{z}$, corresponding to the subset $S_{z} \subset$ П. The center $\mathfrak{z}\left(\mathfrak{g}_{0}\right) \subset \mathfrak{h}$ consists of the elements orthogonal to $S_{z}$. Denote by $R$ the set of roots of $\mathfrak{g}$ and by $R_{z} \subset R$ the set of roots of $\mathfrak{g}_{z}$. Then the nonsingular elements of $\mathfrak{z}\left(\mathfrak{g}_{0}\right)$ that define a nondegenerate bilinear pairing between $\mathfrak{n}_{+}$and $\mathfrak{n}_{-}$are those having nonvanishing scalar product with all elements of $R \backslash R_{z}$. They form a Zariski open subset of $\mathfrak{z}\left(\mathfrak{g}_{0}\right)$. In particular $z$ is a central nonsingular element in $\mathfrak{z}\left(\mathfrak{g}_{0}\right)$.

If $z$ is a regular semisimple element of $\mathfrak{g}$ we return to Example 2.7.

Example 2.9. Let $\mathfrak{g}=H_{n}$ be the Heisenberg Lie algebra generated by $c$ and by $p_{i}, q_{i}, i=1, \ldots, n$, with the only nonvanishing Lie brackets $\left[p_{i}, q_{j}\right]=\delta_{i j} c$. Define the grading by setting $\operatorname{deg}\left(p_{i}\right)=1, \operatorname{deg}\left(q_{i}\right)=-1$ and $\operatorname{deg}(c)=0$. Then $\mathfrak{g}_{0}=\mathbb{C} c$, and any $\chi \in\left(\mathfrak{g}_{0}\right)^{*}$ such that $\chi(c) \neq 0$ is a nonsingular character.

Example 2.10. Let $\mathfrak{g}=$ Vir be the Virasoro algebra with $\mathfrak{g}_{n}=\mathbb{C} L_{n}, n \in \mathbb{Z}, n \neq 0$ and $\mathfrak{g}_{0}=\mathbb{C} L_{0} \oplus \mathbb{C} \mathfrak{c}$ with the Lie bracket $\left[L_{n}, L_{m}\right]=(n-m) L_{n+m}+\delta_{n+m, 0} \frac{n^{3}-n}{12} \mathfrak{c}$. Let $\chi \in \mathfrak{g}_{0}^{*}$ be defined so that $\chi\left(L_{0}\right)=\Delta, \chi(\mathfrak{c})=c$ for some $\Delta, c \in \mathbb{C}$. Then $\chi$ 
defines the pairing $\left(L_{n}, L_{-n}\right)=2 n \Delta+\frac{n^{3}-n}{12} c$ which is nondegenerate for all pairs $\Delta, c \in \mathbb{C}$ with $\Delta+\frac{n^{2}-1}{24} c \neq 0$ for all $n \in \mathbb{Z}_{>0}$.

2.3. Relation to coadjoint orbits. Let $\mathfrak{g}^{*}:=\bigoplus_{i \in \mathbb{Z}} \mathfrak{g}_{i}^{*}$ be the (graded) dual of the Lie algebra $\mathfrak{g}$. It carries a natural coadjoint action of $\mathfrak{g}$. An element $\chi \in \mathfrak{g}_{0}^{*} \subset \mathfrak{g}^{*}$ defines a point in $\mathfrak{g}^{*}$. Our second assumption is equivalent to saying that $\mathfrak{g}_{0}$ is the coadjoint stabilizer of $\chi$.

Assume that $\mathfrak{g}$ is finite dimensional. Then there is a connected simply connected Lie group $G$ with Lie algebra $\mathfrak{g}$ and with a natural coadjoint action of $G$ on $\mathfrak{g}^{*}$. Denote the stabilizer of $\chi$ by $G_{0} \subset G$. It is a closed subgroup of $G$ with Lie algebra $\mathfrak{g}_{0}$. A coadjoint orbit of $\chi$ under the $G$-action is a homogeneous space $M=G / G_{0}$. Our construction of invariant $*$-products applies if the adjoint action of $G_{0}$ preserves the grading. If $G_{0}$ is connected, this condition is automatically satisfied. In particular, this is always the case when $\mathfrak{g}$ is a semisimple Lie algebra.

If $G_{0}$ is disconnected let $H$ be the connected component of the unit element. Then our method applies to $G / H$, which covers the coadjoint orbit $G / G_{0}$.

Let $G$ be a real Lie group and $\mathfrak{g}$ be the corresponding real Lie algebra. Since the $*$ products are usually defined on the space of complex valued functions, it is sufficient that the assumptions of the previous section be satisfied for the complexified Lie algebra $\mathfrak{g}^{\mathbb{C}}$. For this reason, in the rest of the paper we assume that $\mathfrak{g}$ is a complex Lie algebra.

Recall that the coadjoint orbits carry an invariant symplectic form which is constructed as follows. Identify $T_{e H}(G / H) \cong \mathfrak{n}_{-} \oplus \mathfrak{n}_{+}$and define $\omega(u, v):=-\chi([u, v])$. By the assumptions, this bilinear form establishes a duality between $\mathfrak{n}_{-}$and $\mathfrak{n}_{+}$. Let $u_{i}$ and $v_{i}$ be a pair of dual bases in $\mathfrak{n}_{-}$and $\mathfrak{n}_{+}$, respectively. Then the inverse of $\omega$ is the invariant Kirillov-Kostant-Souriau (KKS) Poisson bi-vector on $G / H$, which is equal to $\sum_{i} u_{i} \wedge v_{i}$ at $e H$. An additional constraint which is often imposed on the bi-differential operator $B$ is that the skew-symmetric part of $B_{1}$ be equal to a given Poisson bi-vector. In our case, this condition reads

$$
B_{1}-B_{1}^{t}=\sum_{i} u_{i} \wedge v_{i}
$$

where $B_{1}^{t}$ is a bi-differential operator obtained by exchanging two copies of $U \mathfrak{g} / U \mathfrak{g} \cdot \mathfrak{h}$ in the tensor product. Geometrically, $\mathfrak{n}_{-}$and $\mathfrak{n}_{+}$define two distributions in $T(G / H)$. If we deal with a real Lie group $G$ and the conditions of the previous section are satisfied for the real Lie algebra $\mathfrak{g}$, these distributions give rise to transverse Lagrangian polarizations on the orbit. In general, we get transverse complex polarizations. 


\section{Generalized Verma modules and the Shapovalov pairing}

In this section we recall the notion and basic properties of the Shapovalov pairing and of the associated canonical element in the tensor product of two opposite generalized Verma modules. The details on the generalized Verma modules can be found, for instance, in [Di].

3.1. Generalized Verma modules. Let $\mathfrak{p}_{+} \bigoplus_{i \geq 0} \mathfrak{g}_{i}$ and $\mathfrak{p}_{-}=\bigoplus_{i \leq 0} \mathfrak{g}_{i}$. A central character $\chi$ of $\mathfrak{g}_{0}$ can be given a $\mathfrak{p}_{ \pm}$-module structure by letting $\mathfrak{n}_{ \pm}$act trivially on it. Define the generalized Verma modules by

$$
M^{ \pm}=\operatorname{Ind}_{U \mathfrak{p}_{ \pm}}^{U \mathfrak{g}} \chi \cong U(\mathfrak{g}) \bigotimes_{U \mathfrak{p}_{ \pm}} \chi
$$

In Example 2.7 we recover the Verma module over $\mathfrak{g}$ of highest weight $\chi$, and in Example 2.8 we recover the scalar generalized Verma module induced from the parabolic $\mathfrak{p}_{+}$. By general properties of the induction, $M^{+}$is isomorphic to $U \mathfrak{n}_{-}$as a $U \mathfrak{n}_{--}$ module generated by $v_{\chi}=1 \otimes \chi$. Similarly, $M^{-} \cong U \mathfrak{n}_{+}$as a $U \mathfrak{n}_{+}$-module generated by $v_{\chi}$. Both Verma modules inherit natural gradings from $U \mathfrak{n}_{+}, U \mathfrak{n}_{-}$.

Suppose that $M$ is a $\mathbb{Z}$-graded $U(\mathfrak{g})$-module, and $V$ is any $U(\mathfrak{g})$-module. Then we define the completed tensor product $M \ddot{\otimes} V \equiv \prod_{i \in \mathbb{Z}} M_{i} \otimes V$ as a $\mathbb{Z}$-graded $U(\mathfrak{g})$-module where elements of $U(\mathfrak{g})$ act by comultiplication. In particular, if both $M$ and $N$ are $\mathbb{Z}$-graded $U(\mathfrak{g})$-modules, then $M \check{\otimes} N$ has two $\mathbb{Z}$-gradings. Often it is convenient to preserve both gradings, $M \check{\otimes} N \equiv \prod_{i, j} M_{i} \otimes N_{j}$, where the elements may have infinite length.

Fix a nonsingular character $\chi$ of $\mathfrak{g}_{0}$. For any $\lambda \in \mathbb{C}$ we consider a rescaled character $\chi_{\lambda}:=\lambda \chi$ and define a pairing between $U \mathfrak{n}_{-}$and $U \mathfrak{n}_{+}$which depends on the parameter $\lambda$. Write $U(\mathfrak{g}) \cong U\left(\mathfrak{g}_{0}\right) \oplus\left(\mathfrak{n}_{-} U(\mathfrak{g}) \oplus U(\mathfrak{g}) \mathfrak{n}_{+}\right)$and let $\phi: U(\mathfrak{g}) \rightarrow U\left(\mathfrak{g}_{0}\right)$ be the projection of an element in $U(\mathfrak{g})$ to the first summand along the second. For any $x \in U \mathfrak{n}_{-}, y \in U \mathfrak{n}_{+}$we set

$$
(x, y)_{\lambda}:=\chi_{\lambda}(\phi(S(y) x)),
$$

where $S: U \mathfrak{g} \rightarrow U \mathfrak{g}$ is the antipode of $U \mathfrak{g}$. That is, $S$ is the unique anti-automorphism of $U \mathfrak{g}$ such that $S(x)=-x$ for all $x \in \mathfrak{g}$.

Let $M_{\lambda}^{+} \cong \operatorname{Ind}_{U \mathfrak{p}_{+}}^{U \mathfrak{g}} \chi_{\lambda}$ and $M_{-\lambda}^{-} \cong \operatorname{Ind}_{U \mathfrak{p}_{-}}^{U \mathfrak{g}} \chi_{-\lambda}$ be the generalized Verma modules. The pairing $(\cdot, \cdot)_{\lambda}$ gives rise to a pairing between $M_{\lambda}^{+}$and $M_{-\lambda}^{-}$. Namely, fix generating vectors $v_{\lambda}=1 \otimes \chi_{\lambda} \in M_{\lambda}^{+}$and $v_{-\lambda}=1 \otimes \chi_{-\lambda} \in M_{-\lambda}^{-}$and let

$$
\left(x v_{\lambda}, y v_{-\lambda}\right):=(x, y)_{\lambda} .
$$

This pairing is called the Shapovalov pairing between the generalized Verma modules. It is $U \mathfrak{g}$-invariant in the following sense: $(a u, v)=(u, S(a) v)$ for $u \in M_{\lambda}^{+}$and 
$v \in M_{-\lambda}^{-}$. The modules $M_{\lambda}^{+}$and $M_{-\lambda}^{-}$are irreducible if and only if $(\cdot, \cdot)_{\lambda}: U \mathfrak{n}_{+} \times$ $U \mathfrak{n}_{-} \rightarrow \mathbb{C}$ is nondegenerate. Indeed, if $x \cdot v_{\lambda}$ lies in a proper submodule of $M_{\lambda}^{+}$and $x \in U \mathfrak{n}_{-}$has maximal degree in this submodule, then $(x, y)_{\lambda}=0$ for all $y \in U \mathfrak{n}_{+}$ by the $U(\mathfrak{g})$-invariance of the pairing, and conversely.

Proposition 3.1. Let $\chi$ be a nonsingular character of $\mathfrak{g}_{0}$. Then the pairing

$$
(\cdot, \cdot)_{\lambda}: U \mathfrak{n}_{-} \otimes U \mathfrak{n}_{+} \rightarrow \mathbb{C}
$$

is nonsingular for almost all $\lambda \in \mathbb{C}$.

Proof. Let $\left\{u_{i}\right\}$ be a homogeneous basis in $\mathfrak{n}_{-}, \chi$ a nonsingular character, and let $\left\{v_{i}\right\}$ the dual basis in $\mathfrak{n}_{+}$with respect to the pairing $(u, v)=-\chi\left([u, v]_{0}\right)$. Choose an order of the elements $u_{i}$ in each graded component of $\mathfrak{n}_{+}$, and enumerate the set $\left\{u_{i}\right\}$ by increased grading. Then we have a PBW-type basis in $U\left(\mathfrak{n}_{ \pm}\right)$. In particular, in each graded component $\left(U \mathfrak{n}_{-}\right)_{-n}, n \in \mathbb{Z}_{+}$, there is a basis $\left\{x_{k}^{(n)}\right\}_{k=1}^{N}$ of monomials in $\left\{u_{i}\right\}$. It can be ordered so that the number of factors in $x_{i}^{(n)}$ is greater than or equal to that in $x_{j}^{(n)}$ whenever $i \leq j$. For each element $x_{k}^{(n)}=u_{k_{1}}^{s_{1}} u_{k_{2}}^{s_{2}} \ldots u_{k_{r}}^{s_{r}}$ set $y_{k}^{(n)}=v_{k_{1}}^{s_{1}} v_{k_{2}}^{s_{2}} \ldots v_{k_{r}}^{s_{r}}$. The elements $\left\{y_{k}^{(n)}\right\}$ form a basis in $\left(U \mathfrak{n}_{+}\right)_{n}$. The number $d_{k}=\sum_{i=1}^{r} s_{i}$ is called the length of $x_{k}^{(n)}$. Let $\lambda \in \mathbb{C}$ and consider the $N \times N$ matrix $M^{n}(\lambda)=\left(x_{k}^{(n)}, y_{l}^{(n)}\right)_{\lambda}$. Its elements are polynomials in $\lambda$. The following statements are easy to check:

(a) The order of the polynomial $\left(x_{k}^{(n)}, y_{l}^{(n)}\right)_{\lambda}$ cannot exceed the length of the shortest of the two monomials $x_{k}^{(n)}, y_{l}^{(n)}$.

(b) For two monomials $x_{k}^{(n)}, y_{l}^{(n)}$ of the same length $d_{k},\left(x_{k}^{(n)}, y_{l}^{(n)}\right)_{\lambda}$ is a polynomial of order strictly less than $d_{k}$ unless $l=k$. If $l=k$, then $x_{k}^{(n)}$ and $y_{k}^{(n)}$ have the same number of factors which are dual to each other with respect to the $(\cdot, \cdot)$ pairing.

(c) We have

$$
\left(x_{k}, y_{k}\right)_{\lambda}=\left(\prod_{i=1}^{r}\left(s_{i}\right) !\right) \lambda^{d_{k}}+P_{k}(\lambda),
$$

where $P_{k}$ is a polynomial of order less than $d_{k}$.

The above implies that we can write

$$
M^{n}(\lambda)=D^{n}(\lambda) C(1+O(1 / \lambda)),
$$

where $D^{n}(\lambda)$ is a diagonal matrix with $\left[D^{n}(\lambda)\right]_{k k}=\left(\prod_{i=1}^{r}\left(s_{i}\right) !\right) \lambda^{d_{k}}$, the matrix $C$ 
is constant lower triangular with units in the diagonal, and $O(1 / \lambda)$ is a matrix whose entries are polynomials in $1 / \lambda$ without a constant term. The determinant of $M^{n}(\lambda)$ is a polynomial in $\lambda$ of order $\sum_{k=1}^{N} d_{k}$ with nonzero leading coefficient. Therefore, the matrix $M^{n}(\lambda)$ is invertible for all but a finite number of values of $\lambda \in \mathbb{C}$. The union of zeros of $M^{n}(\lambda)$ for all $n$ is a countable subset of $\mathbb{C}$. This completes the proof.

3.2. Canonical element $\boldsymbol{F}_{\lambda}$. Let $\lambda \in \mathbb{C}$ such that the pairing $(\cdot, \cdot)_{\lambda}$ be nonsingular. Denote by $F_{\lambda} \in U \mathfrak{n}_{-} \check{\otimes} U \mathfrak{n}_{+}$the canonical element corresponding to the pairing. It has the form $F_{\lambda} \in 1+\left(U \mathfrak{n}_{-}\right)_{<0} \check{\otimes}\left(U \mathfrak{n}_{+}\right)_{>0}$. Let $M_{\lambda}^{+} \check{\otimes} M_{-\lambda}^{-}$be a completed tensor product of two irreducible generalized Verma modules with generating vectors $v_{\lambda}$ and $v_{-\lambda}$. Then $F_{\lambda}\left(v_{\lambda} \otimes v_{-\lambda}\right)$ is the canonical element with respect to the Shapovalov pairing. In particular it is $U \mathfrak{g}$-invariant. Choose a generating vector $v \in V_{0}$ in the trivial $U \mathfrak{g}$ module $V_{0} \cong \mathbb{C}$. Then $v \mapsto F_{\lambda}\left(v_{\lambda} \otimes v_{-\lambda}\right)$ defines a $U \mathfrak{g}$-homomorphism $V_{0} \rightarrow M_{\lambda}^{+} \check{\otimes} M_{-\lambda}^{-}$.

Proposition 3.2. The element $F_{\lambda}$ is a meromorphic function of $\lambda$ which is holomorphic at $\lambda=\infty$. The residue of $F_{\lambda}$ at $\lambda=0$ is given by the formula

$$
\operatorname{Res}_{\lambda=0} F_{\lambda}=\sum_{i=1}^{n} u_{i} \otimes v_{i} \in \mathfrak{n}_{-} \check{\otimes} \mathfrak{n}_{+} \subset U \mathfrak{n}_{-} \check{\otimes} U \mathfrak{n}_{+} .
$$

Proof. Using the bases $\left\{x_{k}^{(n)}\right\}$ and $\left\{y_{k}^{(n)}\right\}$ from the proof of Proposition 3.1 one can write the element $F_{\lambda}$ in the following form:

$$
F_{\lambda}=\sum_{n=0}^{\infty} \sum_{k l}\left(M^{n}(\lambda)\right)_{l k}^{-1} x_{k}^{(n)} \otimes y_{l}^{(n)} .
$$

Decomposition (3) implies that the matrix elements $\left(M^{n}(\lambda)\right)_{l k}^{-1}$ are rational functions of $\lambda$, holomorphic at $\lambda=\infty$. Hence, each bi-graded component of $F_{\lambda}$ is a rational function of $\lambda$ holomorphic at $\lambda=\infty$, which amounts to saying that $F_{\lambda}$ is meromorphic and regular at $\lambda=\infty$.

To compute the residue of $F_{\lambda}$ note that for the matrix $C$ of (3) one has $C_{k l}=\delta_{k l}$ for $k, l$ with $d_{k}=d_{l}=1$. This implies the same property for the inverse matrix $C_{k l}^{-1}=\delta_{k l}$ for $k, l$ with $d_{k}=d_{l}=1$. We write

$$
\begin{aligned}
\operatorname{Res}_{\lambda=0}\left(M^{n}(\lambda)\right)_{l k}^{-1} & =\operatorname{Res}_{\lambda=0} \sum_{m}\left(1+O\left(\lambda^{-1}\right)\right)_{l m} C_{m k}^{-1} \lambda^{-d_{k}}\left(\prod_{i} s_{i} !\right)^{-1} \\
& =\operatorname{Res}_{\lambda=0} C_{l k}^{-1} \lambda^{-d_{k}}\left(\prod_{i} s_{i} !\right)^{-1} .
\end{aligned}
$$

This formula shows that the residue vanishes unless $d_{k}=1$. Then, since $C^{-1}$ is lower triangular with $d_{l} \leq d_{k}$ for $l \geq k$ one has $d_{l}=1$ as well. Hence, the only 
matrix elements which have nonvanishing residues are the diagonal entries $k=l$ with $d_{k}=1$, in which case the residue is equal to one. Since the elements with $d=1$ are basis elements in $\mathfrak{n}_{-}$and $\mathfrak{n}_{+}$we obtain (4), as required.

Remark 3.3. Formula (3) implies that the coefficient $\left(M^{n}(\lambda)\right)_{l k}^{-1}$ of the element $x_{k}^{(n)} \otimes y_{l}^{(n)}$ in $F_{\lambda}$ is a rational function in $\lambda$ of order less than or equal to $-d_{k}$. By exchanging the roles of $k$ and $l$ we see that in fact the order is $\leq-\max \left(d_{k}, d_{l}\right)$.

Remark 3.4. In case when $\mathfrak{g}$ is finite dimensional, let $d_{\max } \in \mathbb{Z}_{+}$be such that $\left(\mathfrak{n}_{+}\right)_{l}=0$ for all $l>d_{\max }$. Then we have the following estimate for the length $d_{k}$ of monomials in the basis of $\left(U \mathfrak{n}_{+}\right)_{n}: d_{k} \geq\left[\frac{n}{d_{\max }}\right]$. Therefore in the Taylor series in $1 / \lambda$ for the coefficients of the matrix $\left(M^{n}(\lambda)\right)^{-1}$ the leading term is of order greater than or equal to $\left[\frac{n}{d_{\max }}\right]$. The coefficients of a given order $m$ in $1 / \lambda$ can appear only in matrices $\left(M^{n}(\lambda)\right)^{-1}$ with $n \leq m d_{\max }$. Since each graded component of $U \mathfrak{n}_{+}$is finite dimensional, $F_{\lambda}$ has only finitely many bi-graded components with coefficients of a given order in $1 / \lambda$.

\section{Solutions of the associativity equation}

In this section we relate the Shapovalov pairing with the associativity equation (1) for the pair $\mathfrak{g}, \mathfrak{h}:=\mathfrak{g}_{0}$. In the proof of the associativity equation we shall use $U \mathfrak{g}$-homomorphisms from generalized Verma modules to various completed tensor products. Our argument uses the method introduced in [EV]; a detailed exposition can be found in $[\mathrm{ES}]$.

4.1. $U \mathfrak{g}$-homomorphisms of generalized Verma modules. One useful property of $U \mathfrak{g}$-homomorphisms is given by the following proposition.

Proposition 4.1. Let $M_{\lambda}^{+}$be an irreducible generalized Verma module with generating vector $v_{\lambda}$. Suppose that for a $U \mathfrak{g}$-module $\mathcal{V}$, there exists a $U \mathfrak{g}$-invariant element $z \in M_{\lambda}^{+} \check{\otimes} \mathcal{V}$ such that $z \in v_{\lambda} \otimes w+\left(U \mathfrak{n}_{-}\right)_{<0} \cdot v_{\lambda} \check{\otimes} \mathcal{V}$ for a certain $w \in \mathcal{V}$. Then such element is unique.

Proof. Assuming that an invariant element exists, we will construct it inductively starting from the summand $v_{\lambda} \otimes w$. Let $\left\{y_{k}\right\}$ and $\left\{x_{k}\right\}$ be the bases in $\left(U \mathfrak{n}_{+}\right)_{>0}$ and $\left(U \mathfrak{n}_{-}\right)_{<0}$ which consist of the elements of the bases in each graded component of $U \mathfrak{n}_{+}$ and $U \mathfrak{n}_{-}$constructed in the proof of Proposition 3.1, and ordered by the increased grading. Then we can write $z=v_{\lambda} \otimes w+\sum_{k} x_{k} v_{\lambda} \otimes w_{k}$ for some elements $w_{k} \in \mathcal{V}$. By assumption we have $\Delta\left(y_{k}\right) z=0$ for all $k>0$. Let $y_{l}$ be a basis element of degree 1. Then $\Delta\left(y_{l}\right) z=v_{\lambda} \otimes y_{l} w+\sum_{k} y_{l} x_{k} v_{\lambda} \otimes w_{k}+\sum_{k} x_{k} v_{\lambda} \otimes y_{l} w_{k}$. Since $M_{\lambda}$ 
is irreducible, by construction of the basis there is only one $x_{l}$ (of degree 1) such that $y_{l} x_{l} v_{\lambda}=v_{\lambda}$, and therefore the element $w_{l}=-y_{l} w \in \mathcal{V}$ is uniquely determined.

Now suppose all elements $w_{k}$ are known for $x_{k}$ of degree less than $s$. Let $y_{l}$ be a basis element of degree $s$, then $\Delta\left(y_{l}\right)=y_{l} \otimes 1+1 \otimes y_{l}+\sum_{n} a_{n} y_{n} \otimes y_{n}^{\prime}$ where $\left\{y_{n}\right\}$ and $\left\{y_{n}^{\prime}\right\}$ are elements of the same basis of $\left(U \mathfrak{n}_{-}\right)_{<0}$ of degree less than $s$ and $\left\{a_{n}\right\}$ are complex coefficients. We have

$$
\begin{aligned}
\Delta\left(y_{l}\right)(z)=v_{\lambda} & \otimes y_{l} w+\sum_{k} y_{l} x_{k} v_{\lambda} \otimes w_{k} \\
& +\sum_{k} x_{k} v_{\lambda} \otimes y_{l} w_{k}+\sum_{n} a_{n} \sum_{k} y_{n} x_{k} v_{\lambda} \otimes y_{n}^{\prime} w_{k}=0 .
\end{aligned}
$$

Since $M_{\lambda}$ is irreducible, for each $y_{n}$ there exists only one element $x_{n}$ of the basis of degree less than $s$, such that $y_{n} x_{n} v_{\lambda}=v_{\lambda}$. Similarly, for $y_{l}$ there is only one element $x_{l}$ of degree $s$ such that $y_{l} x_{l} v_{\lambda}=v_{\lambda}$. We obtain the equality

$$
v_{\lambda} \otimes y_{l} w+v_{\lambda} \otimes w_{l}+\sum_{n} v_{\lambda} \otimes a_{n} y_{n}^{\prime} w_{n}+U\left(\mathfrak{n}_{-}\right) v_{\lambda} \otimes \mathcal{V}=0 .
$$

The elements $w_{n}$ corresponding to $x_{n}$ of degree less than $s$ are already known. This determines uniquely the element

$$
w_{l}=-y_{l} w-\sum_{n} a_{n} y_{n}^{\prime} w_{n}
$$

The proof is completed by induction.

Remark 4.2. In case when $\mathcal{V}=V$ is a finite dimensional module, $\check{\otimes}$ is the usual tensor product, the statement follows from the Frobenius reciprocity of the induction:

$$
\operatorname{Hom}_{U \mathfrak{g}}\left(V_{0}, M_{\lambda}^{+} \otimes V\right)=\operatorname{Hom}_{U \mathfrak{g}}\left(M_{-\lambda}^{-}, V\right)=\operatorname{Hom}_{U \mathfrak{p}_{-}}\left(\chi_{-\lambda}, V\right) .
$$

Therefore, the space of $U \mathfrak{g}$-homomorphisms $V_{0} \rightarrow M_{\lambda}^{+} \otimes V$ coincides with the space of $\left(U \mathfrak{n}_{-}\right)$-invariant vectors in $V$ with the action of $U \mathfrak{g}_{0}$ given by the character $\chi-\lambda$.

Proposition 4.3. Let $A: \mathfrak{g} \rightarrow \mathfrak{g}$ be a Lie algebra automorphism of $\mathfrak{g}$ preserving the grading. Then the element $F_{\lambda}$ is invariant with respect to the natural action of $A$ on $U \mathfrak{n}_{-} \ddot{\otimes} U \mathfrak{n}_{+}$.

Proof. Since $A$ preserves the grading, the element $A\left(F_{\lambda}\right)\left(v_{\lambda} \otimes v_{-\lambda}\right) \in M_{\lambda}^{+} \check{\otimes} M_{-\lambda}^{-}$ is of the form $v_{\lambda} \otimes v_{-\lambda}+\left(U \mathfrak{n}_{-}\right)_{<0} \cdot v_{\lambda} \otimes\left(U \mathfrak{n}_{+}\right)_{>0} \cdot v_{-\lambda}$. Since $A$ is a Lie algebra automorphism, $A\left(F_{\lambda}\right)\left(v_{\lambda} \otimes v_{-\lambda}\right)$ is $U \mathfrak{g}$-invariant. Hence, by Proposition 4.1 one has $A\left(F_{\lambda}\right)\left(v_{\lambda} \otimes v_{-\lambda}\right)=F_{\lambda}\left(v_{\lambda} \otimes v_{-\lambda}\right)$, which implies $A\left(F_{\lambda}\right)=F_{\lambda}$. 
Remark 4.4. Assume that $\mathfrak{g}$ can be integrated to a Lie group $G$, and $H \subset G$ is a subgroup with Lie algebra $\mathfrak{g}_{0}$ such that $\operatorname{Ad}_{H}$ preserves the grading. Then the element $F_{\lambda}$ is $\operatorname{Ad}_{H}$-invariant.

Proposition 4.5. Let $\lambda \in \mathbb{C}$ be such that the module $M_{\lambda}^{+}$is irreducible and denote $p: U \mathfrak{g} \rightarrow U \mathfrak{g} / U \mathfrak{g} \cdot \mathfrak{g}_{0}$ the natural projection. Then

$(1 \otimes p \otimes 1)\left[(\Delta \otimes 1) F_{\lambda}\left(F_{\lambda} \otimes 1\right)\right]=(1 \otimes p \otimes 1)\left[(1 \otimes \Delta) F_{\lambda}\left(1 \otimes F_{\lambda}\right)\right]$

in $U \mathfrak{n}_{-} \check{\otimes} U \mathfrak{g} / U \mathfrak{g} \cdot \mathfrak{g}_{0} \check{\otimes} U \mathfrak{n}_{+}$.

Remark 4.6. In case when $\mathfrak{g}$ is semisimple and $\mathfrak{g}_{0}$ is its Cartan subalgebra, equation (5) is a projection from $U(\mathfrak{g})^{\check{\otimes} 3}$ to $U \mathfrak{n}_{-} \check{\otimes} U \mathfrak{g} / U \mathfrak{g} \cdot \mathfrak{g}_{0} \check{\otimes} U \mathfrak{n}_{+}$of the dynamical cocycle equation which was considered in [EV], [EE] and [ESS]. The element satisfying such a condition is called a dynamical twist and is used further to construct solutions of the dynamical Yang-Baxter equation.

For the trivial representation $V_{0}$ of $U\left(\mathfrak{g}_{0}\right)$ define $\mathcal{V}_{0}=\operatorname{Ind}_{U\left(\mathfrak{g}_{0}\right)}^{U(\mathfrak{g})} V_{0}$. Choose a nonzero vector $v \in V_{0}$ and denote $\tilde{v}:=1 \otimes v$ the generating vector in $\mathcal{V}_{0}$.

Lemma 4.7. Let $\lambda \in \mathbb{C}$ be such that the module $M_{\lambda}^{+}$is irreducible.

(a) There exists a unique $U(\mathfrak{g})$-homomorphism

$$
\mathcal{F}_{\lambda}^{+}: M_{\lambda}^{+} \rightarrow M_{\lambda}^{+} \check{\otimes} \mathcal{V}_{0}
$$

such that

$$
\mathcal{F}_{\lambda}^{+}\left(v_{\lambda}\right) \in v_{\lambda} \otimes \tilde{v}+\left(U \mathfrak{n}_{-}\right)_{<0} \cdot v_{\lambda} \otimes U \mathfrak{g} \cdot \tilde{v}
$$

Explicitly,

$$
\mathcal{F}_{\lambda}^{+}\left(v_{\lambda}\right)=F_{\lambda}\left(v_{\lambda} \otimes \tilde{v}\right)
$$

(b) Similarly, there exists a unique $U \mathfrak{g}$-homomorphism

$$
\mathcal{F}_{-\lambda}^{-}: M_{-\lambda}^{-} \rightarrow \mathcal{V}_{0} \check{\otimes} M_{-\lambda}^{-}
$$

such that for a fixed vector $v \in V_{0}$

$$
\mathcal{F}_{\lambda}^{-}\left(v_{-\lambda}\right) \in \tilde{v} \otimes v_{-\lambda}+U \mathfrak{g} \cdot \tilde{v} \otimes\left(U \mathfrak{n}_{+}\right)_{>0} \cdot v_{-\lambda} .
$$

Explicitly,

$$
\mathcal{F}_{-\lambda}^{-}\left(v_{-\lambda}\right)=F_{\lambda}\left(\tilde{v} \otimes v_{-\lambda}\right) .
$$


Proof. Consider the first statement. The element $F_{\lambda}\left(v_{\lambda} \otimes \tilde{v}\right)$ is $U \mathfrak{n}_{+}$-invariant because of the $U \mathfrak{g}$-invariance of the Shapovalov's pairing and the $U \mathfrak{n}_{+}$-invariance of $v_{\lambda}$. The subalgebra $U \mathfrak{g}_{0}$ acts on $F_{\lambda}\left(v_{\lambda} \otimes \tilde{v}\right)$ by the character $\chi_{\lambda}$. Therefore, by the universal property of the generalized Verma module, there is a homomorphism $\mathcal{F}_{\lambda}^{+}: M_{\lambda}^{+} \rightarrow$ $M_{\lambda}^{+} \check{\otimes} \mathcal{V}_{0}$ mapping the generating vector $v_{\lambda}$ to $F_{\lambda}\left(v_{\lambda} \otimes \tilde{v}\right)$. The proof of the uniqueness for a given choice of $v \in V_{0}$ coincides verbatim with the proof of Proposition 4.1. The second statement is proved similarly.

Lemma 4.8. Let $\lambda \in \mathbb{C}$ be such that $M_{\lambda}^{+}$is irreducible. The two morphisms of $U \mathfrak{g}$-modules

$$
V_{0} \rightarrow M_{\lambda}^{+} \check{\otimes} M_{-\lambda}^{-} \stackrel{\mathcal{F}_{\lambda}^{+} \check{\otimes i} \mathrm{id}}{\longrightarrow} M_{\lambda}^{+} \check{\otimes} \mathcal{V}_{0} \otimes M_{-\lambda}^{-}
$$

and

$$
V_{0} \rightarrow M_{\lambda}^{+} \check{\otimes} M_{-\lambda}^{-} \stackrel{\mathrm{id} \otimes \mathcal{F}_{\lambda}^{-}}{\longrightarrow} M_{\lambda}^{+} \check{\otimes} \mathcal{V}_{0} \check{\otimes} M_{-\lambda}^{-}
$$

coincide.

Proof. Both homomorphisms map $v$ to $v_{\lambda} \otimes \tilde{v} \otimes v_{-\lambda}+\left(U \mathfrak{n}_{-}\right)_{<0} \cdot v_{\lambda} \check{\otimes} U(\mathfrak{g}) \cdot \tilde{v} \check{\otimes}$ $\left(U \mathfrak{n}_{+}\right)_{>0} \cdot v_{-\lambda}$. Such a homomorphism is unique by Lemma 4.7 .

Proof of Proposition 4.5. Written in terms of $F_{\lambda} \in \sum_{i}\left(U \mathfrak{n}_{+}\right)_{-i} \otimes\left(U \mathfrak{n}_{-}\right)_{i}$, the equality of the two homomorphisms in Lemma 4.8 gives the statement of the theorem.

4.2. Invariant $*$-products. Denote by $\pi: U \mathfrak{g}^{\otimes 2} \rightarrow\left(U \mathfrak{g} / U \mathfrak{g} \cdot \mathfrak{g}_{0}\right)^{\otimes 2}$ the natural projection. The following theorem is the main result of this paper.

Theorem 4.9. Let $\chi$ be a nonsingular character of $\mathfrak{g}_{0}$ and $F_{\lambda}$ be the corresponding canonical element in $U \mathfrak{n}_{-} \check{\otimes} U \mathfrak{n}_{+}$. Then the element $B:=\pi\left(F_{\hbar^{-1}}\right)$ takes values in $\left(\left(U \mathfrak{g} / U \mathfrak{g} \cdot \mathfrak{g}_{0}\right)^{\otimes 2}\right)^{\mathfrak{g}_{0}}[[\hbar]]$ and satisfies the associativity equation (1).

Proof. Recall that $F_{\lambda}$ is a meromorphic function of $\lambda$ holomorphic at $\lambda=\infty$. Therefore $F_{\mu^{-1}}$ is holomorphic at zero and defines a formal power series $F_{\hbar^{-1}} \in$ $\left(U \mathfrak{n}_{-} \check{\otimes} U \mathfrak{n}_{+}\right)[[\hbar]]$ with projection $B:=\pi\left(F_{\hbar^{-1}}\right) \in\left(U \mathfrak{g} / U \mathfrak{g} \cdot \mathfrak{g}_{0}\right)^{\otimes 2}[[\hbar]]$. The $U \mathfrak{g}$-invariance of the Shapovalov pairing implies the $\mathfrak{g}_{0}$-invariance of $\pi\left(F_{\lambda}\right)$ and as a consequence the $\mathfrak{g}_{0}$-invariance of $B$.

Projecting equation (5) to $\left(U \mathfrak{g} / U \mathfrak{g} \cdot \mathfrak{g}_{0}\right)^{\otimes 3}$ yields the associativity equation for $\pi\left(F_{\lambda}\right)$. Since the latter is holomorphic at infinity, the associativity equation for $B$ follows.

Remark 4.10. As usual, assume that $\mathfrak{g}$ can be integrated to a Lie group $G$ and $\mathfrak{g}_{0}$ can be integrated to a subgroup $H \subset G$ such that $\operatorname{Ad}_{H}$ preserves the grading. Then $\pi\left(F_{\lambda}\right)$ is an element in $\left(\left(U \mathfrak{g} / U \mathfrak{g} \cdot \mathfrak{g}_{0}\right)^{\otimes 2}\right)^{H}[[\hbar]]$ which satisfies the associativity equation. Hence it defines an invariant $*$-product on $G / H$. 
Remark 4.11. In a recent paper [DM2], a categorical approach to constructing dynamical twists is developed. It allows the authors to obtain a quantization of function algebras on semisimple coadjoint orbits for $\mathfrak{g}$ reductive and $\mathfrak{g}_{0}$ its Levi subalgebra. The difference between the approach of [DM2] and the one chosen in the present paper is that we do not use finite dimensional representations of $U \mathfrak{g}$ and harmonic analysis on $G / H$.

Proposition 4.12. Let $\left\{u_{i}\right\}$ and $\left\{v_{i}\right\}$ be dual bases in $\mathfrak{n}_{-}$and $\mathfrak{n}_{+}$with respect to the pairing $-\chi\left([\cdot, \cdot]_{0}\right)$. Then the element $B$ has the form

$$
B=1+\hbar \pi\left(\sum_{i} u_{i} \otimes v_{i}\right)+O\left(\hbar^{2}\right) .
$$

Proof. By Proposition 3.2 the first two terms in the Taylor expansion of $F_{\lambda}$ at $\lambda=\infty$ are as follows:

$$
F_{\lambda}=1+\lambda^{-1} \sum_{i} u_{i} \otimes v_{i}+O\left(\lambda^{-2}\right) .
$$

Replacing $\lambda^{-1} \mapsto \hbar$ and applying projection $\pi$ yields the result.

Remark 4.13. The skew-symmetric part of the first order term in $\hbar$ in the Taylor expansion of $B$ defines an invariant bi-vector on $M=G / H$. Its value at $e H \in M$ is given by $\sum_{i=1}^{n} u_{i} \wedge v_{i}$, which is exactly the KKS bi-vector on the coadjoint orbit.

Remark 4.14. By Remark 3.3 the elements $x_{k} \otimes y_{l}$ which occur with a factor $\lambda^{-n}$ in the element $F_{\lambda}$ have the property $d_{k}, d_{l} \leq n$. Hence, the bi-differential operators $B_{n}$ in the $*$-product $\pi\left(F_{\hbar^{-1}}\right)$ have the order $\leq n$ in each factor. Such $*$-products are called natural. In [GR] it is shown that a natural $*$-product induces a symplectic connection on the underlying manifold $M$. In the case of coadjoint orbits considered in this paper such a connection is defined by a $\mathfrak{g}_{0}$-invariant complement to $\mathfrak{g}_{0}$ in $\mathfrak{g}$, $\mathfrak{n}_{+} \oplus \mathfrak{n}_{-} \subset \mathfrak{g}$.

Example 4.15. Let $\mathfrak{g}=H_{n}$ be the Heisenberg algebra defined in Example 2.9, and let $\chi: c \mapsto w \in \mathbb{C}$ be a nonsingular character, $w \neq 0$. Then the matrix of the Shapovalov pairing in each graded component $\left(U\left(\mathfrak{n}_{-}\right)_{-k}, U\left(\mathfrak{n}_{+}\right)_{k}\right)$ is diagonal, with matrix elements

$$
\left(q_{1}^{k_{1}} \ldots q_{n}^{k_{n}}, p_{1}^{k_{1}} \ldots p_{n}^{k_{n}}\right)_{\lambda}=(-\lambda w)^{k} \prod_{i=1}^{n} k_{i} !
$$

where $k=k_{1}+\cdots+k_{n}$. The corresponding inverse element $F_{\lambda}$ is given by

$$
F_{\lambda}=\sum_{k_{1}, \ldots k_{n}=0}^{\infty} \frac{(-1)^{k}}{(\lambda w)^{k} k_{1} ! \ldots k_{n} !} q_{1}^{k_{1}} \ldots q_{n}^{k_{n}} \otimes p_{1}^{k_{1}} \ldots p_{n}^{k_{n}} .
$$


Using the product structure of $U \mathfrak{n}_{-} \otimes U \mathfrak{n}_{+}$one can write the answer for $B$ in the following compact form:

$$
B=\exp \left(-\frac{\hbar}{w} \sum_{i=1}^{n} q_{i} \otimes p_{i}\right)
$$

This gives a $*$-product on the coadjoint orbit $G / G_{0}=\mathbb{R}^{2 n}$ of the Heisenberg group acting on $\mathfrak{g}^{*}$. It is a 'normal ordered' version of the Moyal product $[\mathrm{M}]$.

Example 4.16. Let $\mathfrak{g}=\operatorname{sl}(2, \mathbb{C})$ with generators $e, f, h$ and Lie brackets $[e, f]=$ $h,[h, e]=2 e,[h, f]=-2 f$. Then $\mathfrak{g}_{0}=\mathbb{C} h$ and $\chi(h)=z \in \mathbb{C}, z \neq 0$ defines a nonsingular character. The element $B$ associated to $\operatorname{sl}(2, \mathbb{C})$ and $\chi$ is given by the formula

$$
B=1+\sum_{n=1}^{\infty} \hbar^{n}\left[\frac{(-1)^{n}}{n ! z(z-\hbar) \ldots(z-(n-1) \hbar)}\right] f^{n} \otimes e^{n}
$$

Remark 4.17. By the theorem of Cahen, Gutt and Rawnsley [CGR], in the case when $\mathfrak{g}$ is semisimple, there are no $*$-products on $\mathfrak{g}^{*}$ with the first order term given by the KKS Poisson bi-vector which restrict to the coadjoint orbits. This is illustrated by the above example: starting from the second graded component, the expression (6) for the $*$-product is singular at $z=0$.

Remark 4.18. Set $G=\mathrm{SU}(2)$. Then the stabilizer of a nonsingular character $\chi \in \mathfrak{g}^{*}$ is isomorphic to $G_{0}=\mathrm{U}(1)$. The homogeneous space $M=G / G_{0}=S^{2}$ is the underlying manifold for the $*$-product constructed above. In this case an explicit formula for the $*$-product similar to (6) appeared before in physics literature (see [P], $[\mathrm{N}]$ and $[\mathrm{HNT}])$.

Example 4.19. Let Vir be the Virasoro algebra defined in Example 2.10 and $\chi$ a nonsingular character. We will use the notation of 2.10 to give an explicit expression for the element $B$, corresponding to Vir and $\chi$, up to the second graded component. The character $\chi$ being nonsingular implies in particular that $\Delta \neq 0$ and $A:=-32 \Delta^{3}-4 \Delta^{2} c \neq 0$. Denote $B:=20 \Delta^{2}-2 \Delta c$, and set $D=A+\hbar B$. Then

$$
\begin{gathered}
B=1-\hbar\left[\frac{1}{2 \Delta}\right] L_{-1} \otimes L_{1}+\hbar\left[\frac{8 \Delta^{2}}{D}+\hbar \frac{4 \Delta}{D}\right] L_{-2} \otimes L_{2}+\hbar^{2}\left[\frac{6 \Delta}{D}\right] L_{-2} \otimes L_{1}^{2} \\
-\hbar^{2}\left[\frac{6 \Delta}{D}\right] L_{-1}^{2} \otimes L_{2}+\hbar^{2}\left[\frac{A}{8 D}\right] L_{-1}^{2} \otimes L_{1}^{2}+\left(U \mathfrak{n}_{-}\right)_{\leq-3} \otimes\left(U \mathfrak{n}_{+}\right)_{\geq 3} .
\end{gathered}
$$


Vol. 80 (2005)

\section{References}

[ABC] D. Arnal, H. Benamor, B. Cahen, Algebraic deformation program on minimal nilpotent orbit. Lett. Math. Phys. 30 (1994), 241-250. Zbl 0805.17009 MR 1267005

[A] A. Astashkevich, On Karabegov's quantizations of semisimple coadjoint orbits. In Advances in geometry, Progr. Math. 172, Birkhäuser, Boston, MA, 1999, 1-18. Zbl 0930.37031 MR 1667673

[AB] A. Astashkevich, R. Brylinski, Non-local equivariant star product on the minimal nilpotent orbit. Adv. Math. 171 (2002), 86-102. Zbl 1010.22021 MR 1933385

[BFFLS] F. Bayen, M. Flato, C. Frønsdal, A. Lichnerowicz and D. Sternheimer, Deformation theory and quantization, I. Deformations of symplectic structures. Ann. Phys. 111 (1978), 61-110. Zbl 0377.53024 MR 0496157

[BBEW] M. Bordemann, M. Brischle, C. Emmrich, S. Waldmann, Phase space reduction for star-products: an explicit construction for $\mathbb{C P}^{n}$. Lett. Math. Phys. 36 (1996), 357-371. Zbl 0849.58035 MR 1384642

[CGR] M. Cahen, S. Gutt, J. Rawnsley, On tangential star products for the coadjoint Poisson structure. Comm. Math. Phys. 180 (1996), 99-108. Zbl 0859.17017 MR 1403860

[Di] J. Dixmier, Algèbres Enveloppantes. Gauthier Villars Éditeur, Paris-BrusselsMontreal, 1974. Zbl 0308.17007 MR 0498737

[DL] M. De Wilde, P. Lecomte, Existence of star-products and of formal deformations of the Poisson Lie algebra of arbitrary symplectic manifolds. Lett. Math. Phys. 7 (1983), 487-496. Zbl 0526.58023 MR 0728644

[DM1] J. Donin, A. Mudrov, Explicit equivariant quantizationon coadjoint orbits of GL $(n, \mathbb{C})$. Lett. Math. Phys. 62 (2002), 17-32. Zbl 1030.53090 MR 1952112

[DM2] J. Donin, A. Mudrov, Dynamical Yang-Baxter equation and quantum vector bundles. Comm. Math. Phys. 254 (2005), 719-760. Zbl 02169977 MR 2126489

[Dr] V. G. Drinfeld, On constant, quasiclassical solutions of the Yang-Baxter quantum equation. Soviet Math. Dokl. 28 (1983), 667-671. Zbl 0553.58038 MR 0725930

[EE] B. Enriquez, P. Etingof, Quantization of Alekseev-Meinrenken dynamical $r$-matrices. In Lie groups and symmetric spaces, Amer. Math. Soc. Transl. (Ser. 2) 210 (54), Amer. Math. Soc., Providence, RI, 2003, 81-98. Zbl 1039.17013 MR 2018354

[EV] P. Etingof, A. Varchenko, Exchange dynamical quantum groups. Comm. Math. Phys. 205 (1999), 19-52. Zbl 0943.17010 MR 1706916

[ES] P. Etingof, O. Schiffmann, Lectures on the dynamical Yang-Baxter equations. In Quantum groups and Lie theory, Cambridge University Press., Lond. Math. Soc. Lecture Note Ser. 290, Cambridge University Press, Cambridge 2001, 89-129. Zbl 1036.17013 MR 1903961

[ESS] P. Etingof, T. Schedler, O. Schiffmann, Explicit quantization of dynamical $r$-matrices for finite dimensional semisimple Lie algebras. J. Amer. Math. Soc. 13 (2000), 595-609. Zbl 0957.17011 MR 1758755

[F] B. Fedosov, A simple geometric construction of defomration quantization. J. Differential Geom. 40 (1994), 213-238. Zbl 0812.53034 MR 1293654 
[G] S. Gutt, An explicit *-product on the cotangent bundle of a Lie group. Lett. Math. Phys. 7 (1983), 249-258. Zbl 0522.58019 MR 0706215

[GR] S. Gutt, J. Rawnsley, Natural star products on symplectic manifolds and quantum moment maps. Lett. Math. Phys. 66 (2003), 123-139. Zbl 02069006 MR 2064595

[HNT] K. Hayasaka, R. Nakayama, Y. Takaya, A New Noncommutative Product on the Fuzzy Two-Sphere Corresponding to the Unitary Representation of SU(2) and the SeibergWitten Map. Phys. Lett. B 553 (2003), 109-118. Zbl 1006.81086 MR 1973356

[Ka] A. V. Karabegov, On deformation quantization on a Kählerian manifold that is related to the Berezin quantization. Funct. Anal. Appl. 30 (1996), 142-144. Zbl 0869.53045 MR 1402088

[K] M. Kontsevich, Deformation quantization of Poisson manifolds. Lett. Math. Phys. 66 (2003), 157-216. Zbl 1058.53065 MR 2062626

[M] J. Moyal, Quantum mechanics as a statistical theory. Proc. Cambridge. Philos. Soc. 45 (1949), 99-124. Zbl 0031.33601 MR 0029330

[N] V. P. Nair, Gravitational fields on a noncommutative space. Nucl. Phys. B 651 (2003), 313-327. Zbl 1008.83021 MR 1957823

[P] P. Presnajder, The origin of chiral anomaly and the non-commutative geometry. J. Math. Phys. 41 (2000), 2789-2804. Zbl 0979.81083 MR 1755469

[T] D. Tamarkin, Fedosov connections on Kähler symmetric manifolds and trace density computation. J. Differential Geom. 50 (1998), 387-413. Zbl 0961.53045 MR 1684985

Received July 15, 2004

A. Alekseev, University of Geneva, Section of Mathematics, 2-4 rue du Lièvre,

1211 Genève 24, Switzerland

E-mail: alekseev@math.unige.ch

A. Lachowska, École Polytechnique Fédéral de Lausanne, SB IMB CAG, Batiment MA C2 604, Station 8, 1015 Lausanne, Switzerland

E-mail: anna.lyakhovskaya@epfl.ch 